\title{
EFFECT OF AN EDUCATIONAL PROGRAM ABOUT GAME STATISTILS AND A GOA-SETTIMG INTERVENTION IN A COMMUNITY COLLEGE WOMEN'S VOLLEYBALL TEAM
}

\author{
José M. Palao \\ Department of Health, Exercise Science and Sport Management. University of Wisconsin - Parkside, United States \\ Address for coprespondence: \\ José M. Palao \\ University of Wisconsin - Parkside \\ SAC D140A. Kenosha, WI 53144, United States \\ E-mail: palaojm@gmail.com
}

\begin{abstract}
Ahstract. The aim was to assess an educational program about game statistics and a goal setting intervention in a community college women's volleyball team. The sample consisted of 12 players from a Midwestern community college women's volleyball team. A quasiexperimental design with a pre-test, post-test, and re-test was done. The dependent variable was the players' perception and use of the game statistics. The independent variable was the educational program about game statistics and goal setting. The coaches' and players' perception, level of satisfaction, and completion of the task during the sessions were recorded. Data were collected through questionnaires and interviews. A descriptive and inferential analysis of the results (t-test for evidence of relationship) was done. After the educational training, the players' perceptions of their ability to understand, analyse, and use information about game statistics and do goal setting in practice and in competition increased significantly. The players used the information significantly more in their practice for all the game actions. Players believed that the abilities they learned helped to improve their performance and the way they practiced. The players believed that the intervention was useful, that it helped them to focus in practice, and that it helped them to focus on technique and the quality of the actions, as well as to better monitor their progress. The educational program was effective with regard to increasing players' perception and understanding about statistics and in the use of statistics and goal setting in practice for monitoring the quality of their actions and their progress.
\end{abstract}

Key Worlds: team sports, performance, coaching, goal-setting, game statistics

\section{Introduction}

In sport, game statistics involve the categorization of the players' actions that are carried out in practice and competition. Usually, the categorization of the actions' efficacy is done in relation to the effect of the actions on the game (error, continuity, or point) or ideal movement pattern (Palao, Morante, 2013). Coding the actions in numbers allows for a mathematical analysis of the data (occurrence, percentages, coefficients, ratios, etc.) (Schleuder, 1998). This allows coaches to carry out a numerical study of the players' and teams' actions in order to make decisions 
based on their abilities. Typically, coaches use game statistics after receiving education about them in their formal training to be coaches. However, players receive information about statistics without receiving training. This risks the players not properly understanding or internalizing this information. In volleyball, the use of game statistics is common to monitor players and teams in practice and in competition (Palao, Hernandez-Hernandez, 2014). In the literature, it is possible to find information about technical-tactical performance indicators (i.e. Mesquita, Palao, Marcelino, Afonso, 2013), reference values (i.e. Peña, Rodríguez-Guerra., Buscà, Serra, 2013), and theory about how to set goals for practice and for competition (Burton, Raedeke, 2008; Weinberg, Butt, 2014).

Several studies have shown the positive effect of goal-setting and the aspects that must be considered with regard to motivation when this psychological technique is used (e.g. Vansteenkiste, Mouratidis, Van Riet, Lens, 2014; Zetou, Papacharisis, Mountaki, 2008). Less is known about the way to introduce players to monitoring their technical and tactical actions and goals or how to train them in this. The numerous actions carried out by the players in practice make it difficult for coaches to monitor players' goals. This could be the reason why coaches and athletes find goal- setting only moderately effective, due to the players' limitation in obtaining feedback about their actions and internalizing it (Burton, Raedeke, 2008). No data are available regarding how players understand and internalize this information or the level of self-monitoring used in their practice and competition. Therefore, although coaches are using this kind of information to guide their decisions and actions, it is not clear whether the terminology, type of calculations, focus, number of criteria, etc. are adapted for their use by the players.

Another aspect to consider is the way this process is implemented within the teams. Teams usually have a specific member or several members of the coaching staff who do the statistics. This person is the one who carries out the data collection, analysis, and data report. However, there is no information regarding how players monitor their actions in practice and competition, if they do. Educational training could be a tool to introduce and enhance the utility and productivity of game statistics in sport. This could help players to be more aware of the important aspects of the game, to know which actions they have to work on, and to see which aspects they should emphasize in practice and competition in the long-term. This could help the coaching process be more centered on the athlete (Hendry, Hodges, 2013; Vansteenkiste et al., 2014). The aim was to assess the educational program about game statistics and the goal setting intervention in a community college women's volleyball team.

\section{Method}

The sample consisted of 12 players from a U.S. Midwestern community college women's volleyball team (2012-2013 season). The sample had an average age of $18.8 \pm 1.7$ years, height of $1.71 \pm 0.07$ meters, weight of $65.52 \pm 8.00 \mathrm{~kg}$, and experience of $7.2 \pm 2.1$ years of playing volleyball. The coaching staff was composed of a head coach, two assistant coaches, and a strength and conditioning coach. The characteristics of the coaching staff were an average age of $38.8 \pm 3.7$ years and an average experience of $10.2 \pm 4.6$ years coaching volleyball. The players usually stayed in the program two years. One of the assistant coaches performed the team statistics as required by the competition's organizing committee. Her functions and data collection protocols were set by the team's conference and the National Collegiate Athletic Association (2012). The sample was selected due to accessibility. The coaches and the athletic department of the college accepted the invitation to participate in the study. All players were informed of the study and its goals. They completed a consent form before starting the educational training. The educational training was integrated as part of the training done by the team. 
A quasi-experimental design with a pre-test, post-test, and re-test was done. The dependent variable was the players' perception and use of the game statistics. The independent variable was the educational program about game statistics and goal setting. The intervention program was carried out in the pre-season, and the follow-up was carried out during the competitive season (14 training weeks). Also, the coaches' and players' perception and level of satisfaction about the educational program was monitored, as was the completion of the tasks done by the players during the sessions. Data were collected through questionnaires and interviews. The program had four 30 -minute theoretical-practical sessions outside of the gym and three sessions of 30 minutes in the gym. Players were taught how to set goals and monitor their actions. A wall poster was used to monitor the team goals related to game statistics.

The educational training attempted to give the players the ability to know: what are game statistics?, what types of calculation can be done?, how are data evaluated?, and how can I use game statistics to improve? The structure of the theoretical-practical sessions outside of the gym was 15 minutes of information presented with the use of slides, video, and combining information with examples; 10 minutes of an applied task (e.g. how to read a match report, how to interpret data, how to monitor and register an action from a video, etc.); two minutes of multiple choice questions to evaluate the knowledge acquisition; and three minutes to review these questions. The structure of the practical sessions in the gym was 3-4 minutes of task explanations, 2-3 series of 4-8 minutes to perform the task, and 3-4 minutes of monitoring the actions, recording the information, linking the qualitative and qualitative execution, and reviewing the process.

During the sessions, players were taught to evaluate their action's efficacy in practice and to extract the information from the match report. Individual and collective goals were set by the players. From the analysis of the previous season's statistics (statistics of the team, the other conference teams, and teams that attended the National Championship), reference values were set (Tables 1 and 2). When establishing the players' values, player positions were taken into consideration (Table 3). In order to connect the quantitative monitoring with improving a certain technique, a set of key aspects were given to the players to review their technical execution when they did not achieve their goals. The key aspects were determined by the coaches and the researcher in order to maintain the same correction criteria in the feedback that the players received in practice. Self-record sheets were used to set the individual goals and to monitor the actions during practices and register information from match reports. The team goals were placed on a poster. Coaches placed stickers on the poster after each game. After the

Tahle 1. Reference values for terminal actions and actions of continuity by team (data from the winning team of every match from the previous season of the community college athletic conference that was studied, 2010-2011 season)

\begin{tabular}{|c|c|c|c|c|c|c|}
\hline \multirow{2}{*}{ By Set } & \multicolumn{2}{|c|}{ Serve } & \multicolumn{2}{|c|}{ Attack } & \multicolumn{2}{|c|}{ Block } \\
\hline & occurrence & percentage & occurrence & percentage & occurrence & percentage \\
\hline Errors & $1-2$ per set & $<10$ & 5 per set & $<15$ & 1-2 per set & $<10$ \\
\hline Continuity & & & 15 per set & 45 & & \\
\hline \multirow[t]{2}{*}{ Point } & 2-3 per set & $>10$ & 14 per set & $>40$ & 2-3 per set & $>10$ \\
\hline & \multicolumn{2}{|c|}{ Reception } & \multicolumn{2}{|c|}{ Assist } & \multicolumn{2}{|c|}{ Digs } \\
\hline Errors & 1 per set & 5 & & & & \\
\hline Continuity & 1 per set & 5 & & & & \\
\hline Perfect & 18 per set & 90 & 11 per set & 35 & 18-19 per set & $>50$ \\
\hline
\end{tabular}

To obtain players' values, divide by number of players involved in these actions on your team.

Teams achieved 25 points through points obtained from the serve, attack, block, and opponent errors (which accounted for 6-7 points). 
educational training, the team integrated the monitoring into practices. Coaches promoted its use but did not force it. The team goals were monitored during the entire season.

Table 2. Reference values for terminal actions and actions of continuity (U.S. National Community College Championship, 2010-2011 season)

\begin{tabular}{|c|c|c|c|c|c|c|}
\hline \multirow{2}{*}{ By Set } & \multicolumn{2}{|c|}{ Serve } & \multicolumn{2}{|c|}{ Attack } & \multicolumn{2}{|c|}{ Block } \\
\hline & occurrence & percentage & occurrence & percentage & occurrence & percentage \\
\hline Errors & 1 per set & $<10$ & 4 per set & $<12$ & $1-2$ per set & $<10$ \\
\hline Continuity & & & 14 per set & $<40$ & & \\
\hline \multirow[t]{2}{*}{ Perfect } & 3 per set & $>10$ & 17 per set & $>50$ & 3 per set & $>10$ \\
\hline & \multicolumn{2}{|c|}{ Reception } & \multicolumn{2}{|c|}{ Assist } & \multicolumn{2}{|c|}{ Digs } \\
\hline Errors & 1 per set & 5 & & & & \\
\hline Continuity & 1 per set & 5 & & & & \\
\hline Perfect & 18 per set & 90 & 14 per set & $>40$ & 20 per set & $>60$ \\
\hline
\end{tabular}

To obtain players' values, divide by number of players involved in these actions on your team.

Teams achieved 25 points through points obtained from the serve, attack, block, and opponent errors (which accounted for 4-5 points).

Table 3. Theoretical importance of the different actions in relation to player position

\begin{tabular}{ll}
\hline Position & Theoretically most important aspects \\
Outside hitter & Serve (errors), reception (errors), hit percentage and digs (total) \\
Middle blocker & Serve (errors), hit percentage, blocks (total), and digs (total) \\
Opposite & Serve (errors), hit percentage, blocks (total), and digs (total) \\
Setter & Serve (errors) and assists \\
Libero & Serve (errors), reception (errors), and digs (total) \\
\hline
\end{tabular}

The questionnaires used to collect the data were designed ad hoc and they were validated by two experts in sport performance, coaching, or goal-setting (content validity). The questionnaires evaluated the players' perception of the game statistics and their use. The experts and the head coach reviewed the questionnaires and evaluated the adequacy, pertinence, and vocabulary. After the educational training, the level of perception and knowledge of the players about game statistics was measured. After the season, an interview was carried out with the players and the head coach. The interviews were done by the researcher using video-conference. The interview questions focused on the effects of the educational training and the skills learned on their engagement, understanding, and usage, as well as an overall evaluation of the process. The researcher performed the analysis of the qualitative data on the interview transcripts and the resulting themes for the purposes of reporting. A descriptive and inferential analysis of the quantitative results (Wilcoxon Test for evidence of relationship) was done.

\section{Results}

After the educational training, players' perception of the importance of the statistics for the public and media increased significantly (Table 4). Their perception of the importance of the statistics for coaches and players did not change (initial and final values $>9.5$ out of 10 ). Players' perceptions of their ability to understand, analyse, and use statistics in practice and in competition increased significantly. The players used the information significantly more 
in their practices for all the game actions. Players believed that the abilities they learned helped to improve their performance and the way they practiced. The players believed that the intervention was useful, because it helped them with technique and the quality of their actions in practice, as well as to better monitor their progress. In relation to the educational training, the players' average involvement was adequate for sessions outside of the gym (65\% task achievement) and good for sessions in the gym (>70\% task achievement).

Table 4. Players' perception of the effect of skills learned (values expressed on a scale from 0 to 10 and by percentage)

\begin{tabular}{|c|c|c|c|c|c|}
\hline & \multirow{2}{*}{\multicolumn{2}{|c|}{ Before }} & \multirow{2}{*}{\multicolumn{2}{|c|}{ After }} & \multirow{3}{*}{$\mathrm{p}$} \\
\hline & & & & & \\
\hline & Mean & SD & Mean & SD & \\
\hline Importance for coaches & 9.91 & 0.30 & 10.00 & 0.00 & 0.315 \\
\hline Importance for players & 9.30 & 1.06 & 9.60 & 0.97 & 0.460 \\
\hline Importance for media & 6.73 & 1.95 & 8.40 & 1.71 & 0.024 \\
\hline Importance for public & 5.27 & 2.28 & 8.20 & 1.75 & 0.017 \\
\hline Understanding & 6.20 & 1.99 & 8.80 & 0.79 & 0.005 \\
\hline Analyzing & 5.50 & 2.12 & 8.40 & 1.51 & 0.005 \\
\hline Applying to practices & 5.50 & 1.43 & 8.60 & 1.17 & 0.005 \\
\hline Applying to competition & 6.10 & 1.97 & 8.50 & 1.35 & 0.005 \\
\hline Level of use & 5.40 & 1.99 & 9.00 & 1.15 & 0.026 \\
\hline Serve & 7.60 & 1.90 & 9.30 & 1.06 & 0.017 \\
\hline Pass & 6.20 & 1.75 & 8.80 & 1.40 & 0.005 \\
\hline Set & 5.10 & 2.13 & 8.10 & 1.79 & 0.005 \\
\hline Attack & 6.60 & 2.88 & 9.10 & 1.29 & 0.010 \\
\hline Block & 6.40 & 2.95 & 8.50 & 1.43 & 0.017 \\
\hline Dig & 6.50 & 1.84 & 9.10 & 1.10 & 0.005 \\
\hline Improved performance (\%) & - & - & 100.00 & 0.00 & - \\
\hline Improved practice (\%) & - & - & 91.90 & 0.32 & - \\
\hline
\end{tabular}

From the analysis of the interviews, three general themes emerged that summarized the players' and head coach's responses to the intervention. These are listed as (a) increased knowledge and skills, (b) change in players' perspective of the way to practice, and (c) more awareness regarding their actions.

a) Increased knowledge and skills. The educational training involved an increase in the players' perceptions of their ability to understand, interpret, and use statistics. During the interviews, players commented:

"...l look more at the numbers and use them in practice" [Player \#2] "...now it's part of daily training" [Player \#6]

b) Changing players' perspective of the way to practice. The educational training affected the way players executed their practice, as well as their desire to work harder. During the interviews, the players and head coach commented:

"It really hasn't affected my involvement much, but it has influenced the way I practice" [Player \#2], "Now I compete with myself and try to improve my stats every day" [Player \#1], "Makes you work harder in practice" [Player \#10], "Gives sense to practice, monitoring our work, work-out... Really helps us as a team" [Head Coach]. 
Specifically about the poster, they commented:

"...part of daily training" [Player \#2]; "Gave us team bonding time" [Player \#8], and "High impact... we wanted more stickers" [Player \#1].

c) More awareness regarding their actions. The educational training involved an increase in the players' perceived ability to understand, interpret, and use the information and goal setting. During the interview, the players and head coach commented:

"It makes me realize that I have to work really hard during practice" [Player \#3], "It makes me focus on the right thing during practice" [Player \#4], and "They realize where they are and that they had to work really hard to achieve their goals" [Head Coach].

About the poster, they specifically commented:

"It helped us to figure out what we should be working on in our next practice" [Player \#2]; "It made me focus on the right thing during practice" [Player \#4]; "The poster changed our goals" [Player \#10]; and "It helps the players realize where we were and what we have to achieve in order to be successful" [Head Coach].

\section{Discussion}

The goal of the present study was to assess the effect of an educational program about game statistics and a goal-setting intervention on a sport team. In the interpretation of the data, the facts that the data came from one team and there was no control group should be taken into consideration. This case study is a first step in the development of educational training protocols for monitoring players' technical and tactical actions. Data show that the educational program was effective for increasing players' perception regarding their understanding of statistics, as well as their usage in practice to monitor their quality and progress. The results suggest that this could be considered a step to increase player participation in the coaching process (Hendry, Hodges, 2013).

Players indicated that the intervention increased their knowledge and understanding, their ability to analyse the data, and their ability to apply the skills learned in practice. The increase in knowledge and understanding seems logical because most of the players had not had previous training about these skills, and during the intervention they obtained specific training and individual and collective feedback. The absence of a control group does not allow us to know about the possible effect of an expectancy on the player's perception (Thomas, Nelson, Silverman, 2011). The educational training was determined to be positive and useful by players. The training program that was tested complements the proposal for developing goal-setting skills proposed by Burton, Raedeke (2008). The characteristics of team sports involve the need for players to self-monitor during practice. In addition to providing information, the tested protocol involved acquiring and implementing a goal-setting process, providing information about the technical and tactical performance indicators, and how to monitor and use this process to evaluate themselves.

This educational training involved the players learning to monitor their actions and specific common tasks, such as analysing box scores or match reports or monitoring their actions during practice. All this individual feedback was combined with collective feedback (match report and poster) from the coaching staff. Thus, the training that was done involved the same activities that players had to do during a normal week: play a match, analyse the data obtained from the game (match report), monitor their actions in practice, try to improve, and so on. Therefore, the protocol was tested in real situations and with experienced athletes. Another aspect that must be considered regarding the intervention is that the performance goals (action efficacy) of the different actions were connected to 
process goals, key indicators of the actions. The objective, besides monitoring themselves, was to involve players in their actions, and to make them reflect on what they have to pay attention to or change. This combination of individual and team monitoring about process and performance aspects allows us to obtain measurable goals of their actions that they can use in practice (Burton, Raedeke, 2008).

Indirectly, the findings showed that the information and skills provided to the players was adequate for their level, and they understood and used them. The data were expressed to the players in ratios expressed in relation to 10 (e.g. three kills of 10 attempts) (Palao, 2008; Schleuder, 1998). This mathematical expression was selected in order to make it easier for players to monitor their actions. Therefore, although coaches manage game statistics using percentage, occurrence per set, ratios, or coefficients, the information was always presented to the players in ratios to increase the chances of players applying the data in their practice. The integration of applied theory with the normal, everyday practice of the athletes could also be a key aspect of the results found with this team. More research is needed about the language coaches should use to communicate with their athletes in order to enhance this process (Kristiansen, Tomten, Hanstad, Roberts, 2012).

From the comments of the players, the educational program also contributed to an increased involvement of the players or the way that players carried out their practice. The reason for this may be due to players having reference values to achieve individually and collectively, players having the ability to monitor themselves, or players participating in the process of establishing their individual and team technical and tactical goals. These results coincide with the effects or benefits of goal setting found by Burton and Raedeke (2008). This educational training works with players that have seven years of experience. The sample studied had automated the techniques, so they were able to have an external focus during their technical executions. With a sample of community college players, the skills that the players learned allowed them to challenge themselves. The combination of the possibility of obtaining information, feedback, and having reference values is probably the reason for the "awareness" that the players and the head coach commented on in the interviews. Knowing their level, their evolution, and the level needed to achieve the national competition makes them realize where they are and how much work is needed to achieve their goals. This correction of their self-perception is a normal adjustment caused by the original lack of skills to properly estimate their level (Kruger, Dunning, 1999). Previous research about the effect of goal setting has found that although its use is positive with younger players (Zetou et al., 2008), with more experienced players, this effect is not as clear, probably due to the number of aspects that affect their performance (Goldsmith, 2008; Hopkins, 2004).

For the team that participated in the study, the effect of the educational training was positive, and it helped the coaches give their athletes meaning to the practices and work-outs. The proposal increased the involvement of players in their training process by giving them skills to monitor their actions and reference values to evaluate these actions (O'Donoghue, Mayes, 2013). This knowledge can increase their awareness of their actions, their procedural knowledge, and their engagement in the process (Hendry, Hodges, 2013). Also, it will allow better integration for the players in the monitoring process of the training, which allows them to be more involved in the process, even more autonomous, and less dependent on the game-to-game dynamic (Vansteenkiste et al., 2014). Previous studies have shown the positive effect of reflective mentoring by coaches on elite players' procedural knowledge, decision making, and performance (Moreno, Moreno, Urena, Iglesias, Villar, 2008). However, it is not clear whether these abilities increase during the formation process (Araujo, Afonso, Mesquita, 2012). This experience introduces the possibility that systematic and deliberate theoretical-practical training for players of the skill of self-monitoring their 
actions (game statistics) could help to develop players' awareness about their actions and affect the procedural knowledge, decision making, and performance. Future studies are needed to confirm these findings in teams of different levels, gender, age groups, etc., with the use of a control group, and while assessing the effect on the quality of the practice and player improvement.

\section{Conclusions}

The educational program increased players' perception and understanding of statistics as well as their use in practice to monitor the players' quality and progress. The findings show that teaching players to monitor and evaluate their actions in practice and in competition increases the involvement of the players and the awareness of their needs. The steps and materials developed in this study can be used as a reference for the way to introduce these knowledge and skills to players. The study involved providing knowledge and skills about how to monitor the efficacy of their actions, the aspects of the execution to review if the efficacy level was not achieved, and reference values to analyse their data, as well as how to set collective and individual goals. More studies are needed to assess the proper vocabulary, tasks, protocols, etc. to adapt theory to practice and to the level of the players.

\section{Réerenences}

Araújo, R.M.F., Neves, J.A., Mesquita, I.M.R. (2012). Procedural knowledge, decision making and performance in women's volleyball according to age group and specific experience. Open Sports Sciences Journal, 5, 167-173. DOI: 10.2174/1875399X01205010167.

Burton, D., Raedeke T.D. (2008). Sport psychology for coaches. Champaign, IL: Human Kinetics.

Goldsmith, W. (2008, February 1st). Making sense of testing athletes. Sports Coaching Brain Blog [Web log message]. Retrieved from http://www.sportscoachingbrain.com/making-sense-of-testing-athletes.html.

Hendry, D.T., Hodges, N.J. (2013). Getting on the right track: athlete-centred for expert performance. In: T. McGarry, P. O'Donoghue, J. Sampaio (eds.), Routledge Handbook of Sports Performance Analysis (pp. 5-20). London: Routledge.

Hopkins, W.G. (2004). How to interpret changes in an athletic performance test. Sportscience, 8, 1-7.

Kristiansen, E., Tomten, S.E., Hanstad, D.V., Roberts, G.C. (2012). Coaching communication issues with elite female athletes: Two Norwegian case studies. Scandinavian Journal of Medicine \& Science in Sports, 22 (6), e156-e167. DOI: 10.1111/j.1600-0838.2012.01521.x.

Kruger J., Dunning, D. (1999). Unskilled and unaware of it: how difficulties in recognizing one's own incompetence lead to inflated selfassessments. Journal of Personality and Social Psychology, 77 (6), 1121-1134.

Mesquita, I., Palao, J.M., Marcelino, R., Afonso, J. (2013). Chapter 29. Indoor volleyball and beach volleyball. In: T. McGarry, P. O'Donoghue, J. Sampaio (eds.), Routledge Handbook of Sports Performance Analysis (pp. 367-379). London: Routledge.

Moreno, M.P., Moreno, A., Ureña, A., Iglesias, D., Villar F.D. (2008). Application of mentoring through reflection in female setters of the Spanish national volleyball team. A case study. International Journal of Sport Psychology, 39 (1), 59-76.

National Collegiate Athletic Association (2012, July 15). NCAA Official volleyball statistics rules. Approved rulings and interpretations. Retrieved from http://www.ncaa.org/championships/statistics/ncaa-volleyball-statisticians-manuals.html.

O'Donoghue, P., Mayes, A. (2013). Performance analysis, feedback and communication in coaching. In: T. McGarry, P. O'Donoghue, J. Sampaio (eds.), Routledge Handbook of Sports Performance Analysis (pp. 155-164). London: Routledge.

Palao, J.M., Morante, J.C. (2013). Technical effectiveness. T. McGarry, P. O'Donoghue, J. Sampaio (eds). Routledge Handbook of Sports Performance Analysis (pp. 213-224). London: Routledge.

Palao, J.M., Hernández-Hernández, E. (2014). Game statistical system and criteria used by Spanish volleyball coaches. International Journal of Performance Analysis in Sport, 14 (2), 564-573.

Peña, J., Rodríguez-Guerra, J., Buscà, B., Serra, N. (2013). Which skills and factors better predict winning and losing in high-level men's volleyball? The Journal of Strength \& Conditioning Research, 27 (9), 2487-2493. DOI: 10.1519/JSC.0b013e31827f4dbe.

Schleuder, S. (1998). Comprehensive volleyball statistics. A guide for coaches, media and fans. Arkansas City, AK: American Volleyball Coaches Association. 
Thomas, J.R., Nelson, J.K., Silverman S.J. (2011). Research methods in physical activity. Champaign, IL: Human Kinetics.

Vansteenkiste, M., Mouratidis, A., Van Riet, T., Lens W. (2014). Examining correlates of game-to-game variation in volleyball players' achievement goal pursuit and underlying autonomous and controlling reasons. Journal of Sport Exercise Psychology, 36 (2), 131-145. DOI: 10.1123/jsep.2012-0271.

Weinberg, R., Butt, J. (2014). Goal-setting and sport performance. In: A.G. Papaioannou, D. Hackfort (eds.), Routledge companion to sport and exercise psychology: global perspectives and fundamental concepts (pp. 343-355). London: Routledge.

Zetou, E., Papacharisis, V., Mountaki, F. (2008). The effects of goal-setting interventions on three volleyball skills: a single-subject design. International Journal of Performance Analysis in Sport, 8 (3), 79-95.

Cite this article aS." Palao J.M. (2016). Effect of an Educational Program about Game Statistics and a Goal-Setting Intervention in a Community College Women's Volleyball Team. Central European Journal of Sport Sciences and Medicine, 14 (2), 27-35. DOI: 10.18276/cej.2016.2-04. 
